PROCEEDINGS OF THE

AMERICAN MATHEMATICAL SOCIETY

Volume 30, No. 1, September 1971

\title{
THE FAILURE IN COMPUTABLE ANALYSIS OF A CLASSICAL EXISTENCE THEOREM FOR DIFFERENTIAL EQUATIONS
}

\author{
OLIVER ABERTH
}

\begin{abstract}
An example is given of a uniformly continuous constructive function $f(x, y)$ with $f(0,0)=0$, such that the differential equation $y^{\prime}=f(x, y)$ with the initial condition $y(0)=0$ has no constructive solution.
\end{abstract}

Introduction. In the recent articles [1], [2] we gave a development of an analysis over a subfield of the reals, the computable numbers. This analysis is equivalent to the constructive analysis investigated by the Russian mathematicians Markov, Ceitin, Zaslavskir, Sanin, and their followers, who have obtained many interesting and fundamental results over the past two decades. (See, for instance, [4], [5], $[6],[8],[11],[12],[13],[15],[17]$ and [18].) Their definitions and constructions ultimately involve the concept of "normal algorithm," or less frequently, the concept of "recursive function." In our development the concept of "program" plays the central role.

The object of this paper is to show, in computable analysis, that there is a function $f(x, y)$, uniformly continuous in a rectangle $R$ centered at the origin, such that the differential equation

$$
y^{\prime}(x)=f(x, y(x))
$$

has no solution function $y(x)$ satisfying the initial condition $y(0)=0$. There is no solution, no matter how small an interval for $x$ about 0 is prescribed, or whether 0 is an interior point, left endpoint, or right endpoint of the interval. This contrasts with the situation in real analysis where, by a theorem of Peano (see [14], [10, pp. 59-66]), if $f(x, y)$ is uniformly continuous in a rectangle $R$ and the initial condition is appropriate, there always exists a solution function satisfying (1), although there is known no generally applicable constructive method for finding a solution. The failure of Peano's Theorem in computable analysis may be interpreted as showing that no such constructive method is possible.

The equation $y^{\prime}=f(x, y)$. Our first theorem is valid in both real

Presented to the Society, January 24, 1971 under the title $A$ nonexistence theorem of differential equations; received by the editors June 24, 1970.

AMS 1969 subject classifications. Primary 0270, 0275, 0280.

Key words and phrases. Computable analysis, constructive function, computable number, program, Peano's Theorem, derivative, Lipschitz condition. 
analysis and computable analysis, with different interpretations, however, for many of the expressions used.

THEOREM 1. If the function $f(x, y)$ is uniformly continuous in a rectangle $R:|x-a| \leqq M_{1},|y-b| \leqq M_{2}$, and if $f(x, y)$ satisfies the inequalities $|f(x, y)| \leqq M_{2} / M_{1},\left|f\left(x, y_{1}\right)-f\left(x, y_{2}\right)\right| \leqq L\left|y_{1}-y_{2}\right|$, for points $(x, y),\left(x, y_{1}\right),\left(x, y_{2}\right)$ in $R$, and $L$ a positive number, then there exists $a$ unique uniformly continuous function $y(x), x$ in the interval $|x-a|$ $\leqq M_{1}$, which satisfies (1) and the initial condition $y(a)=b$.

In computable analysis the terms "number" (i.e. "computable number"), "function", "uniformly continuous function", and "derivative" have constructive definitions. In our notation these are given by Definitions $8,15,22$, and 24 resp. of [1]. Our definition of the derivative of $f(x)$ at $x_{0}$ resembles

$$
\lim _{x_{1}, x_{2} \rightarrow x_{0} ; x_{1} \neq x_{2}} \frac{f\left(x_{1}\right)-f\left(x_{2}\right)}{x_{1}-x_{2}} \text { instead of } \lim _{x_{1} \rightarrow x_{0}} \frac{f\left(x_{1}\right)-f\left(x_{0}\right)}{x_{1}-x_{0}} .
$$

However, the theorems of this paper are valid for either version of derivative definition. The derivative of the function $y(x)$ of Theorem 1 at the endpoints of the interval $|x-a| \leqq M_{1}$ is to be interpreted, of course, as an appropriate right-hand or left-hand derivative. Also, the assertion of the existence of $y(x)$ is meant in a strong sense, that is, there is an effective method of finding $y(x)$ (see [1, p. 286]). The constructive methods of Cauchy-Lipschitz [9, pp. 75-80], or of successive approximations [3, pp. 99-115], are readily modified to obtain a proof of this theorem in computable analysis, and we omit the details. We require later a version of Theorem 1 where the point $(a, b)$ is not at the center of the rectangle $R$ but at its left or right side. Thus, if in the definition of $R$ the range $|x-a| \leqq M_{1}$ is replaced by $0 \leqq x-a \leqq M_{1}$, the conclusion still holds if the interval domain for $y(x)$ is similarly altered.

The next theorem gives our main result.

THEOREM 2. There exists a function $f(x, y)$, uniformly continuous in the rectangle $R:|x| \leqq 1,|y| \leqq 1$, and with $|f(x, y)| \leqq 1$ for $(x, y)$ in $R$, such that for any interval $[a, b]$ which is a subset of $[-1,1]$ and contains the point 0 , there is no function $y(x)$ defined in the interval which satisfies (1) and the initial condition $y(0)=0$.

We shall continue to employ the terminology of [1]. For a program $P$, the rational number $P(a)$ and the positive integer $N_{P}$, the "descriptive integer of $P$," were specified in Definitions 1 and 3 of that article. First we prove a useful 
Lemma. There is no programmable function $g(n)$ which equals \pm 1 and is defined for positive integers $n$ greater than a fixed integer $n_{0}$, such that $g(n)=+1$ if $n=N_{P}$ with $P\left(N_{P}\right)$ defined and not positive, $g(n)=-1$ if $n=N_{P}$ with $P\left(N_{P}\right)$ defined and positive.

Suppose otherwise. Let $Q$ be a program realizing the function $g(n) \quad(Q(n) \equiv g(n))$, with sufficient redundant computation steps added, if necessary, so that $N_{Q}>n_{0}$. Then $Q\left(N_{Q}\right)$ is defined, and whichever of the values \pm 1 it assumes leads to a contradiction.

For the proof of Theorem 2 we shall construct the function $f(x, y)$ so that it has the following additional three properties:

(i) $f(x, y)=-f(-x, y)$.

(ii) If $y(x)$ is a solution to (1) for $x$ in the interval $\left[-2^{-n+1},-2^{-n}\right]$, $n \geqq 1$, then $y\left(-2^{-n+1}\right)=y\left(-2^{-n}\right)$.

(iii) If $y(x)$ is a solution to (1) for $x$ in the in terval $\left[-2^{-n+1},-2^{-n}\right]$, $n \geqq 1$, such that $y\left(-2^{-n+1}\right)=0$, then, if $n=N_{P}$ and $P\left(N_{P}\right)$ is defined, at the midpoint of the interval this solution is greater than $2^{-3(n+2)}$ if $P\left(N_{P}\right)$ is positive, and less than $-2^{-3(n+2)}$ if $P\left(N_{P}\right)$ is not positive.

These properties of $f(x, y)$ rule out a solution to (1) near $x=0$ and satisfying the initial condition $y(0)=0$. For suppose $y(x)$ is such a solution in the interval $[a, 0], a<0$. Then we may find a positive integer $n_{0}$ such that $a<-2^{-n_{0}}$. By (ii), $y\left(-2^{-n_{0}}\right)=y\left(-2^{-n}\right)$ for all $n>n_{0}$, and this requires $y\left(-2^{-n_{0}}\right)=0$ since $y(x)$ is continuous and equals 0 at $x=0$. But, by (iii), we may then obtain a function $g(n)$ of the type excluded by the lemma if we define $g(n)$ as -1 if a computed rational approximation to $y\left(\frac{1}{2}\left(-2^{-n+1}-2^{-n}\right)\right)$ with error less than $2^{-3(n+2)}$ comes out positive, and as +1 if the approximation does not come out positive. Thus a solution to the left of the point $x=0$ is not possible. A solution to the right is also not possible, for if $y(x)$ satisfies (1) on the interval $[0, b], b>0$, with $y(0)=0$, by (i), the function $y_{1}(x)=y(-x)$ will be a solution to the left of 0 in the interval $[-b, 0]$ with $y_{1}(0)=0$.

For the definition of $f(x, y)$ we require first the construction of two families of computable functions: $h_{n}(x)$ and $j_{n}(x, y)$.

In [1] there was defined the programmable function $U(n, m, a)$ which equals 1 if $n=N_{P}$ and $P(a)$ is defined after the execution of no more than $m$ computation steps; otherwise $U(n, m, a)=0$. Setting $q(x)$ equal to $\max (1-|x|, 0)$, define the functions $h_{n}(x)$ by the equation

$$
h_{n}(x)= \pm \sum_{k=1}^{\infty}(U(n, k, n)-U(n, k-1, n)) 2^{-k} q\left(2^{k}\left(x-\frac{1}{2}\right)\right)
$$

Thus if $n$ is not the descriptive integer of a program, or if $n=N_{P}$ but 
$P\left(N_{P}\right)$ is undefined, then $h_{n}(x)=0$. If $n=N_{P}$ and $P\left(N_{P}\right)$ is defined, the sign of $h_{n}(x)$ in (2) is taken as + if $P\left(N_{P}\right)>0$ and - otherwise. Hence when $P\left(N_{P}\right)$ is defined in exactly $t$ computational steps, then these signs are definitely assumed by $h_{n}(x)$ inside an open interval of length $2^{-t+1}$ centered at $x=\frac{1}{2}$.

Before constructing $j_{n}(x, y)$ we require some information on certain solutions to the equation $y^{\prime}=s(x, y)$, where $s(x, y)=9 x(1-x) y^{1 / 8}$. It is easy to verify that the equation $y^{\prime}=s(x, y)$, with the initial condition $y(0)=y_{0}$, has in the interval $[0,1]$ for $x$ the solution

$$
y=\left(\operatorname{sgn} y_{0}\right)\left[x^{2}(3-2 x)+\left|y_{0}\right|^{2 / 3}\right]^{3 / 2},
$$

for $y_{0} \neq 0$. The function sgn $t$ equals 1 if $t>0$, and -1 if $t<0 . s(x, y)$ satisfies a Lipschitz condition for $(x, y)$ in any rectangle $R$ for which $|y| \geqq e, e$ a positive number, so these solutions are unique by Theorem 1 . When $y_{0}=0$, there is a family of solutions over $[0,1]$ :

$$
\begin{array}{ll}
y=0, & 0 \leqq x \leqq c, \\
y= \pm\left[x^{2}(3-2 x)-c^{2}(3-2 c)\right]^{3 / 2}, & c \leqq x \leqq 1 .
\end{array}
$$

$c$ may be any number satisfying the inequalities $0 \leqq c \leqq 1$. At the point $x=1$, no two of the solutions given in (3) and (4) have identical values, and there is no number not assumed by one of these solutions at $x=1$. Also, if a solution is unequal to 0 at the point $x_{0}$ in $[0,1)$, it is clear from the differential equation that the solution must increase in modulus in the interval $\left[x_{0}, 1\right]$, and by Theorem 1 there can be no other solution in this in terval with the same value at $x=1$. Combining these details, it is easily shown that there is no solution over $[0,1]$ distinct from those given by (3) and (4).

Now we define the functions $j_{n}(x, y)$ by

$$
\begin{array}{ll}
j_{n}(x, y)=h_{n}(x), & x \in[0,1], \\
j_{n}(x, y)=s(x-1, y), & x \in[1,2], \\
j_{n}(x, y)=-s(x-2, y), & x \in[2,3], \\
j_{n}(x, y)=-h_{n}(x-3), & x \in[3,4],
\end{array}
$$

and

$$
j_{n}(x, y)=0, \quad x \notin[0,4] .
$$

If $y(x)$ is a solution over the interval $[0,4]$ to the equation $y^{\prime}=j_{n}(x, y)$, then by the preceding paragraph, the value of $y(2)$ determines the solution in the interval $[1,2]$, and then certainly in $[0,1]$ also. Using the identities $h_{n}(x)=h_{n}(1-x)$ and $s(x, y)=s(1-x, y)$, it is easy to verify that $j_{n}(x, y)=-j_{n}(4-x, y)$. This implies that the 
function $y_{1}(x)=y(4-x)$ is also a solution in $[0,4]$. Since $y_{1}(2)=y(2)$, $y_{1}(x)=y(x)$, at least in the interval $[0,2]$, and the general equation $y(x)=y(4-x), x$ in $[0,4]$, follows. Thus if $y(x)$ is a solution in $[0,4]$, then $y(0)=y(4)$. In addition, if $y(0)=0$, then if $n=N_{P}$ and $P\left(N_{P}\right)$ is defined, $y(1)>0$ if $P\left(N_{P}\right)>0$, and $y(1)<0$ of $P\left(N_{P}\right) \leqq 0$. This requires $y(2)>1$ if $P\left(N_{P}\right)>0$ and $y(2)<-1$ if $P\left(N_{P}\right) \leqq 0$ (cf. (3)).

Finally, we define $f(x, y)$ for $x \leqq 0$ by the equation

$$
f(x, y)=\sum_{n=1}^{\infty} 2^{-2(n+2)} j_{n}\left(2^{n+2}\left[x+2^{-n+1}\right], 2^{3(n+2)} y\right),
$$

and for other $x$ by requiring $f(x, y)=-f(-x, y)$. Note that under the transformation $\hat{x}=2^{n+2}\left[x+2^{-n+1}\right], y=2^{3(n+2)} y$, a solution to $d \hat{y} / d \hat{x}$ $=j_{n}(\hat{x}, \hat{y}), \hat{x} \in[0,4]$, becomes a solution to

$$
\frac{d y}{d x}=2^{-2(n+2)} j_{n}\left(2^{n+2}\left[x+2^{-n+1}\right], 2^{3(n+2)} y\right)
$$

for $x$ in the interval $\left[-2^{-n+1},-2^{-n}\right]$. Moreover, for $x$ outside this interval the right-hand side of (5) equals 0 . The three properties of $f(x, y)$ listed earlier now follow and the proof is complete.

\section{REFERENCES}

1. O. Aberth, Analysis in the computable number field, J. Assoc. Comput. Mach. 15 (1968), 275-299. MR 38 \#5626.

2. - A chain of inclusion relations in computable analysis, Proc. Amer. Math. Soc. 22 (1969), 539-548. MR 39 \#3998.

3. G. Birkhoff and G. Rota, Ordinary differential equations, Introduction to Higher Math., Ginn, Boston, Mass., 1962. MR 25 \#2253.

4. G. S. Ceitin, Algorithmic operators in constructive metric spaces, Trudy Mat. Inst. Stekov. 67 (1962), 295-361; English transl., Amer. Math. Soc. Transl. (2) 64 (1967), 1-80. MR 27 \#2406.

5. - Mean-value theorems in constructive analysis, Trudy Mat. Inst. Stekov. 67 (1962), 362-384. (Russian) MR 27 \#2407.

6. - Three theorems on constructive functions, Trudy Mat. Inst. Stekov. 72 (1964), 537-543. (Russian) MR 34 \#5669.

7. J. Cleave, The primitive recursive analysis of ordinary differential equations and the complexity of their solutions, J. Comput. System Sci. 3(1969), 447-455. MR 40 \#4512.

8. O. Demut, Necessary and sufficient conditions for the Riemann integrability of constructive functions, Dokl. Akad. Nauk SSSR 176 (1967), 757-758=Soviet Math. Dokl. 8 (1967), 1176-1177. MR 37 \#65.

9. E. L. Ince, Ordinary differential equations, reprint, Dover, New York, 1956.

10. E. Kamke, Differentialgleichungen reeller Funktionen, Chelsea, New York, 1947. MR 8, 514.

11. B. A. Kušner, Riemann integration in constructive analysis, Dokl. Akad. Nauk SSSR 156(1964), 255-257 = Soviet Math. Dokl. 5(1964), 628-630. MR 29 \#22. 
12. A. A. Markov, On the continuity of constructive functions, Uspehi Mat. Nauk 9 (1954), no. 3 (61), 226-230. (Russian) MR 16, 436.

13. —_, On constructive mathematics, Trudy Mat. Inst. Steklov. 67 (1962), 8-14. (Russian) MR 27 \#3528.

14. G. Peano, Démonstration de l'intégrabilité des équations différentielles ordinaires, Math. Ann. 37 (1890), 182-228.

15. N. A. Šanin, Constructive real numbers and constructive functional spaces, Trudy Mat. Inst. Steklov. 67 (1962), 15-294; English transl., Transl. Math. Monographs, vol. 21, Amer. Math. Soc., Providence, R.I., 1968. MR 28 \#30.

16. A. M. Turing, On computable numbers, with an application to the Entscheidungsproblem, Proc. London Math. Soc. (2) 42 (1936/37), 230-265.

17. I. D. Zaslavskir, Some properties of constructive real numbers and constructive functions, Trudy Mat. Inst. Steklov. 67 (1962), 385-457; English transl., Amer. Math. Soc. Transl. (2) 57 (1966), 1-84. MR 27 \#3519.

18. I. D. Zaslavskir and G. S. Ceitin, Singular coverings and properties of constructive functions connected with them, Trudy Mat. Inst. Steklov. 67 (1962), 458-502. (Russian) MR 27 \#2408.

Texas A \& M University, College Station, Texas 77843 